\title{
Barriers for virtual assessment of structural robustness
}

Nerenst, Tim Brix; Ebro, Martin; Nielsen, Morten; Eifler, Tobias; Nielsen, Kim Lau

Published in:

Conference Proceedings - International Mechanical Engineering Congress \& Exposition

Link to article, DOI:

10.1115/imece2019-11251

Publication date:

2020

Document Version

Peer reviewed version

Link back to DTU Orbit

Citation (APA):

Nerenst, T. B., Ebro, M., Nielsen, M., Eifler, T., \& Nielsen, K. L. (2020). Barriers for virtual assessment of structural robustness. In Conference Proceedings - International Mechanical Engineering Congress \& Exposition (Vol. 2B: Advanced Manufacturing). [ IMECE2019-11251] American Society of Mechanical Engineers. https://doi.org/10.1115/imece2019-11251

\section{General rights}

Copyright and moral rights for the publications made accessible in the public portal are retained by the authors and/or other copyright owners and it is a condition of accessing publications that users recognise and abide by the legal requirements associated with these rights.

- Users may download and print one copy of any publication from the public portal for the purpose of private study or research.

- You may not further distribute the material or use it for any profit-making activity or commercial gain

- You may freely distribute the URL identifying the publication in the public portal 


\section{DRAFT: BARRIERS FOR VIRTUAL ASSESSMENT OF STRUCTURAL ROBUSTNESS}

\author{
Tim Brix Nerenst \\ Section of Solid Mechanics \\ Department of Mechanical Engineering \\ Technical University of Denmark \\ Kgs. Lyngby, 2800, Denmark \\ Email:TBNE@MEK.DTU.DK
}

\author{
Martin Ebro \\ Morten Nielsen \\ Engineering analysis \& materials \\ Novo Nordisk \\ Hilleroed, 3400 \\ Denmark
}

\author{
Kim Lau Nielsen \\ Tobias Eifler \\ Department of Mechanical Engineering \\ Technical University of Denmark \\ Kgs. Lyngby, 2800 \\ Denmark
}

\begin{abstract}
Simulation techniques such as Finite Element Analysis (FEA) have substantially contributed to reducing the time and cost of developing new mechanical products over the last decades. However, FEA is primarily carried out on the nominal geometry, i.e. not taking into account the variation in geometrical and material parameters that originates from either the production or use-cases. This can lead to either overly conservative or overly optimistic designs, which in turn leads to unnecessary cost and/or unforeseen product failures. Despite the introduction of commercial FEA tool packages (such as 3DX/Abaqus/ANSYS etc.) that enables designers and engineers to combine FEA and Robust Design (RD) methods, performing sensitivity studies to identify robustness issues is rarely conducted in a systematic manner. The objective of this study is to identify the barriers for why the combined use of FEA and robust design techniques are not a standard part of the design process. The study concludes that combining FEA and RD will require a substantial effort, however create significant value and enhance the design process.
\end{abstract}

\section{NOMENCLATURE}

FEA Finite Element Analysis

DoE Design of Experiments

RD Robust Design
RDO Robust Design Optimization

VRDO Virtual Robust Design Optimization

RDM Robust Design Methodology

CAD Computer Aided Design

CAE Computer Aided Engineering

ES Expert Statements

PS Physical Tests

AS Analytical Solution

VS Virtual Simulation

FR Functional Requirement

\section{INTRODUCTION}

Development of complex products, such as a new medical injection device is a long and difficult process. From the patients perspective, the device must be easy to use and provide the require amount of medicine reliably. High reliability can be achieved if the design is insensitive towards all types of variance during its lifetime, i.e. from production, use-cases, and environment. This is referred to as a robust design.

During the development of a new device, design decisions are made every day to achieve the desired robust functionality. These decisions can be based on expert statements, physical tests, analytical solutions or virtual simulations. Each category has their own strengths and weaknesses in regards to accuracy, cost, speed of use, etc. 


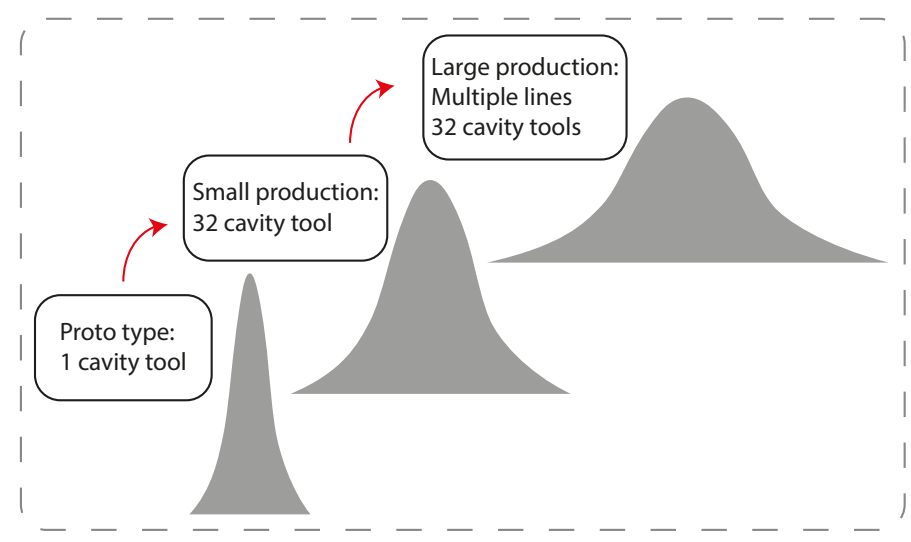

FIGURE 1. VARIATION IMPACT DUE TO PRODUCTION SCALING

Today Robust Design Methodology (RDM) and Finite Element Analysis (FEA) are frequently utilized in large companies aiming for optimal robustness. However, even in leading industry companies such as this papers medical device case company, these methods are rarely used in combination. Even-though the company has substantial R\&D resources and access to high-end software solutions and skilled/specialized employees, still does not systematically apply variation in their analysis assisted by FEA. There seems to be a significant untapped potential in using simulations to predict the performance of the full sample space of a product, i.e. virtually "manufacture" e.g. 10.000 product samples which would represent actual variation cases and predicting their performance.

For the medical device case company robustness issues are predominantly identified and solved during the design and validation process. However, even with the immense effort that is put into applying robust design methodology, some robustness issues are not detected before the production is started. Figure 1 exemplifies how variance can increase as production scales. Knowing the expected variance is key for correct analysis and utilizing prototype builds can hide robustness issues. Valuable learnings in the early design phase could be gained if the effects of variance can be estimated for the most significant design parameters.

As stated in the EU founded paper [1, p. 8] enhancements to virtual development most be industrialized to replace conventional methods of design for continuously identify new and improved designs and avoid limiting innovation. This is further stressed as the acceptable performance window for high-end products is continuously narrowed to satisfy the level of actual and perceived quality. To achieve this performance, the products must be increasingly robust towards variance in geometry, material properties and external factors. Traditionally Robust Design, e.g. kinematic design, P-diagram, tolerance chains, etc. and Fi-

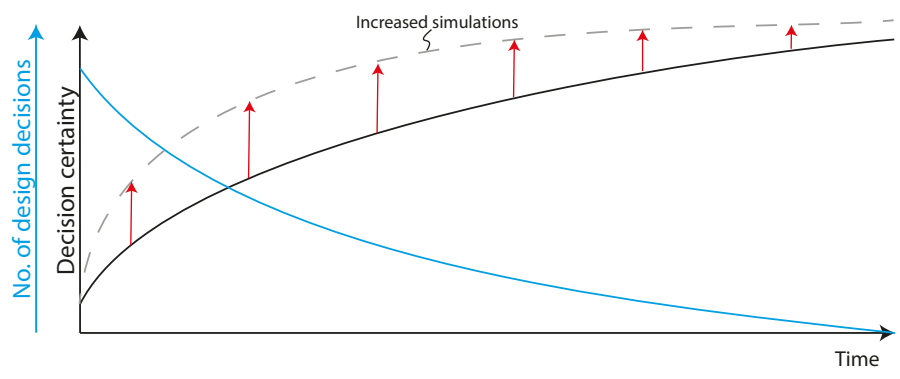

FIGURE 2. PROBLEMATIC RELATIONSHIP BETWEEN PROJECT TIME AND DECISION CERTAINTY.

nite Element Analysis are used separately. Typically one project engineer will apply robust designs methods and another validate nominal failure criteria via FEA. The purpose of this work is to identify the key barriers that hinder the symbiotic use of FEA and RD methods in an industrial context, as it is expected to improve the efficiency and accuracy of robustness detection during a development project. FEA can enable new ways of exploring the full sample space by applying methods such as Design of Experiments (DoE), Sensitivity Analysis, and meta/surrogatemodelling combined with Monte-Carlo on a purely virtual design. The symbiotic use of FEA and RD methods will be referred to as Virtual Robust Design Optimization (VRDO).

It is further theorized that VRDO enables an improvement to the unfortunate inverse relationship between the number of design decisions and the decision certainty as seen in figure 2 . This would be especially impactful in the early phases of development as most design decisions are made early and can be very costly to alter later in the design process.

Decision certainty is defined as the confidence level for a given design decision based on the available information. Ideally a designer strive to have all information available when making a decision, but the reality is that decisions are typically forced through on a limited information basis due to project deadlines [2]. It is the authors opinion that VRDO is the cheapest method for improving the decision certainty. A segment of the increased decision certainty via VRDO would be insight into failure probability distributions instead of using safety factors. This could effectively replace the nominal failure criteria and instead provide a distribution such as part per million expected failures for a defined range of variances. This would also strongly resonate with the risk management which is critical for the medical sector.

The application of Robust design methodology consists of three main principles [3, p. 654];

1. Achieve insensitivity to variation

2. Awareness of variation

3. Beneficial in all design stages

The first statement is the optimal goal, however this will not be 
achieved without the two latter. Awareness of variation is increasing but the tools applicability in all design stages lack behind [3, p. 655].

The goal is to investigate the industrial barriers for enabling VRDO in all phases of the design process. It is theorized that all barriers can be grouped as below and can assist in identifying possible solutions;

1. Technical barriers, e.g. software capabilities, cloud computing, process automation, etc.

2. Practical barriers, e.g. time constraints, limited no. of specialists, parameterized on models, etc.

3. Knowledge barriers, e.g. knowledge of variance impact, sources of variance, etc.

Depending on the category, the solutions might vary anywhere from collaborating with software suppliers, Change management or active teaching in the new methods.

\section{METHODOLOGY}

The empirical data has been gathered from two main sources; an industry leading medical manufacturing company and a software development company. The section is divided as shown below;

1. Manufacturing company

Interview form

Description of the company

Description of interviewees

\section{Software company}

Interview form

Description of the company

Description of interviewee

During the interviews all answers were captured in written form and the accuracy confirmed by the interviewee. Afterwards a systematic review of the data was performed to identify patterns, themes and outliers.

The interview form used with the manufacturing company followed the interview guide/unstructured interview [4, p. 271] and divided the guiding questions into three scenarios. The unstructured interview form was chosen as it provides a format where a discussion around complex topics can be guided and allow the interviewer to explore interesting areas of the conversation. Surveys and closed questions where avoided as the goal was to explore the interviewees personal impression of today's utilization of RD and FEA. The three scenarios where each controlled by a 20 minute window where visual graphs and figures were used to steer the interview and ensure all topics where covered. Scenario 1 revolves around the pros and cons of the cur- rently used decision making tools; Expert statements (ES), Physical tests (PS), analytical solutions (AS), virtual simulation (VS). Scenario 1 is used to create the baseline for which parameters the VRDO should be able to compete with. Scenario 2 revolves around the interviewee giving examples of where the impact of variance has been an issue and caused a negative impact on the design process. Scenario 2 provides the potential value of having improved VRDO tools to evaluate and understand the impact of variance early in the design process. Scenario 3 revolves around the functionality of the VRDO tools. The interviewee is first asked to suggest tools/functionalities which would be helpful during the design process. Secondly, suggestions from the author is discussed. It was also discussed where in the general design process [5, p. 19] the tools would be applicable and create the most value.

The manufacturing company designs and manufactures small hand-held medical injection devices. Due to the large scale of production the standard design guidelines for tolerances is set to IT grade 13 (IT13). IT grades are commonly used for plastic injection molding and defined in equation 1. An example of the expected linear tolerance window can be seen in table 1 .

$$
T=10^{0.2 \times(I T G-1)} \times(0.45 \times \sqrt[3]{D}+0.001 \times D)
$$

\begin{tabular}{ccc}
\hline Nominal & Lower limit & Upper limit \\
\hline 10 & 9.754 & 10.246 \\
\hline
\end{tabular}

TABLE 1. EXAMPLE OF IT13

Using an IT13 as a standard design criteria ensures that the manufacturing costs are controlled and directly provides the initial variance window which the design should manage. However, the tolerance window only defines the acceptable parts from the production facility. It does not ensure the robustness of the design. The aim for the design team should be to produce a design which is robust enough to perform reliably with an IT13 grade or higher. If a lower IT grade is required to avoid robustness issues it would impact the cost of manufacturing by increasing scrap rates and/or tightening of the manufacturing process controls. Integration of robust design methods in the product development has therefore been on-going for the last decade in the company. The company is considered a frontier within the field of robust design and aims to further their development towards VRDO.

The manufacturing respondents are divided into two groups. Group A consists of high-level management deci- 
sion makers. They were selected based on their role in the company as they evaluate project material in milestone meetings. The material presented in said meetings consists of metrics describing the performance of the design, technical risks, and identified issues, along with plans for mitigating these. Finally, they will decide whether the project will should continue, be postponed, or be terminated. The group consisted of three people. Group B consists of Lead Engineers. They were selected based on their role in the company as they take the daily decisions on design, validation, risk management, and are expert users of robust design methods. They also carry the responsibility of presenting the project material at the milestone meetings. The lead engineers are responsible for the design of the device and they interact with a team ranging from 5 to 20 engineers, depending on the project, and they have the option to utilize additional support-functions such as material and finite element specialists. The group consisted of six people. The two groups are chosen based on their direct involvement of future use of VRDO and their knowledge of the current barriers between Robust Design methods and use of FEA. Group A will provide input on what design information could be improved and how the future results should be presented to make efficient high-level decisions. Group B will provide input on current barriers making the use of VRDO inefficient and how they visualize the future design process should utilize the symbiotic effects of RD and FEA. Some bias is inevitable and can impact the information provided during the interviews. This can be expressed by an unwillingness to provide examples where mistakes were made by the interviewee to avoid looking flawed. The interviewees might suggest/try to change the future solutions to maximize their own ease of work, not considering the broader benefit for the company. This is evaluated when examining and concluding on their responses. Also, the results from the company respondents might not reflect the whole industry's view on the subject.

The interview form used with the software company was a closed quantitative interview/structured interview [4, p. 271], where the interviewee ranked statements from strongly disagree to strong agree on a questionnaire. The interview aimed to highlight industry issues seen from a software company and to understand the expected "pull" from the industry for new and improved software capabilities in the field of Virtual Robust Design Optimization. A structured interview was chosen as the responses where used to either directly confirm or deny investigated hypotheses barriers.

The software company develops add-on software with capabilities for DoE, sensitivity \& Robustness studies integrated directly into SOLIDWORKS. Their product aims to assist engineers to optimize their products towards robustness. They wish to achieve this without complicating the design process signifi- cantly by integrating CAD, FEA, and process capabilities, such as automated DoE studies.

The software respondent is the CEO of the company. The respondent has years of experience within the field of software development and the robust design optimization. The respondent provided input on the current industry "pull" and expectation to new software capabilities in the market of virtual robust design optimization.

\section{THE FUTURE OF INDUSTRIAL VIRTUAL DEVELOP- MENT}

The gathered empirical data is split into four sub-sections. The order of the presented data; (1) Pros \& cons of existing product development tools used today, (2) Industrial implications of uncontrolled variation, (3) Ideas for future VRDO tools and processes and finally (4) a list of confirmed and invalidated barriers for further implementation of VRDO in an industrial setting.

\section{Pros \& Cons of existing design decision tools}

Four main categories of design decision making tools are analyzed; expert statements, physical tests, analytical solutions, virtual simulations. Lastly, a visualization of their current contributions in the different phases of product development is presented.

Expert statement is defined as the guidance/decisions made from a person with prolonged experience through education or experience within a field. All lead engineers agree that expert statements are essential in a design process, particularly in the early phase. It is the most effective way of getting a design or concept started and avoid commonly known design faults. For example to provide the initial dimensions/relative sizes for known mechanisms. However, expert statements heavily reply on actual experience. More than one stated that this can result in limited leaps in innovation as the uncertainty greatly increases by doing things differently. For example if a new mechanism or new technology is incorporated in the next generation of a product family, then only relying on expert statements will often not be sufficient to deliver a robust and reliable product. The high-level decision makers also recognize the value in expert statements. Both from their own use and in trusting the lead engineers. However, when complex issues are flagged, more accurate validation methods, such as simulation, are requested to confirm possible solutions and to de-risk the project.

Physical tests is defined as tests performed with physical parts, sub-assemblies or complete products. All lead engineers utilize and recognize the value in physical testing. It can be an effective method for; validating functions in sub-assemblies, calibrating simulations or performing final functional validation testing for the authorities. Sub-assembly testing was mentioned 
as an important method for understanding new mechanisms and it can be performed relatively quick. However, 4 out of 6 highlighted that testing isolated sub-assemblies can hide potential interaction issues in the complete product and can therefore lead to false-positives of a working design. Testing the complete product was mentioned by all as very time-consuming and it is limited to a few experts to perform high quality repeatable tests. However, final testing is always required in the late design stages. 2 out of 6 believed that physical testing was used too much as the primary tool and often without a clear test plan. Their opinion was instead that these efforts should be placed in achieving analytical solutions to understand whether a concept will work, rather than testing sporadically. An outlier statement was that a complete prototype was sometime used to convince the high-level decision makers that a concept was working. The LE regarded this as highly risky as a prototype can be "made to work" by fitting and greasing the parts, however for large scale, fully automated production, sensitivity issues can make the design invalid. Another LE highlighted that not all things can be simulated and that physical testing therefore sometimes is the only method for achieving insight into complex behaviors.

Analytical solutions is defined as solutions to closed-form expressions, for example the maximum deflection of a cantilever beam. All lead engineers utilized analytical solutions to identify whether a design had any potential and to estimate the initial dimensions. However, it differed if the lead engineers thought the method was quick and easy to use. The positive argument was that approximate dimensions could be established for mechanisms if the forces could be estimated and the geometry could be simplified to fit the expressions. The counter-argument was that applying analytical solutions on simplified geometry required additional assumptions which made the calculations complicated and a specialists job. If the simplification was made poorly it could generate a high risk of losing important geometry that would alter the final results. However, all agreed that if analytical solutions could be established, applying Monte-Carlo methods on-top was an excellent way of getting a feel for the sensitivity of the design. This highlighted the existing awareness of variation in the organization. One of the high-level decision makers also highlighted, that no physical tests should be performed before an analytical solution of the problem was established. Otherwise the experimental results would have minimal value as the expected outcome of the test cannot be anticipated and compared with.

Virtual simulations is defined as virtual model that approximates physical behavior. All lead engineers recognized virtual simulations as an essential tool during the design process. In the company two levels of virtual simulations are created. The simpler of the two is performed in the CAD software by a limited number of designers. This is mainly used for initial stress calculations. The advanced virtual simulations are performed by a team of specialists, who support across the projects/organization. 2 out of 6 lead engineers mentioned that the simpler virtual simu- lations created excellent value and suggested that more designers should possess this ability. This method was especially useful for checking whether a design change had improved the design, meaning that even if the simulation was not $100 \%$ accurate, the designer could still evaluate if changes were in the right direction. The main issue with designers utilizing the simpler virtual simulations were the frequency of use. If a project in the company took a total of four years, the designer could spend two years using CAD daily and the last two years working with documentation (consequence of the medical industry). This results in a relearning period once shifted to the next project. By then, the belief in own abilities to use and produce accurate results had often disappeared, and the safer option would therefore be to contact the specialist group. The impression from the lead engineers was that the specialist group provides excellent and accurate results requested by the projects. However, the time-constraint was mentioned as the main barrier. This was further worsened if the simulations should capture the effect of variance, as variation had to be manually introduced. Both the lead engineers and high-level decision makers agreed, that the time-constraint was the main cause to why simulations where mainly used in the late design stages, and not utilized early and on multiple design suggestions. The final and important issue with simulation was highlighted by one of the lead engineers; "It's easy to simulate a known issue, however it is difficult to identify unthought-of issues". The statement meant that it is easy to evaluate whether a stress level in a structure exceeds the material properties if this is the goal of the simulation. It is however very difficult to identify possible misuse scenarios or unexpected contacts if the applied boundary conditions are aimed to investigate another functionality.

Contribution of decision tools can be seen in figure 3 . The top section defines the generic phases of product design [5, p. 19]. The middle section defines the tasks, e.g. functional requirements, creating design solutions, iterations and finally product documentation. The bottom section is the resulting current utilization of the different decision making tools. Critical comments from all company interviewees were that the use of numerical simulations in the early/principle solution phase was too limited.

\section{Industrial implications of uncontrolled variation}

The information gathered for this section can be sensitive for the case company. The results of the conducted interviews are therefore covered in anecdotal form without making the claim to be a complete picture of potential implications. To respect the company's integrity all examples are furthermore rewritten to avoid targeting specific persons or design examples.

When discussing product issues it is critical to differentiate between a failure caused by an unrobust design or by a design failure. The automotive industry describes the differences by the 


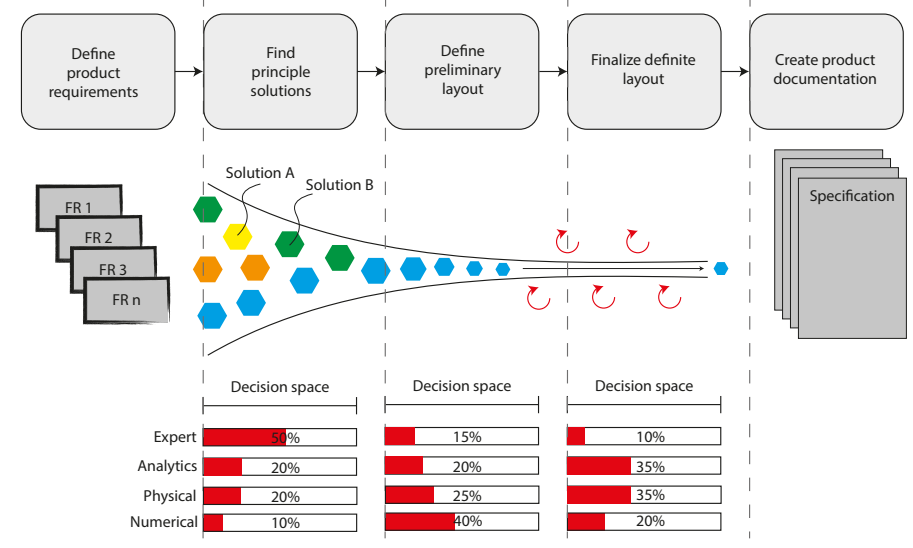

FIGURE 3. CURRENTLY APPLIED TOOLS

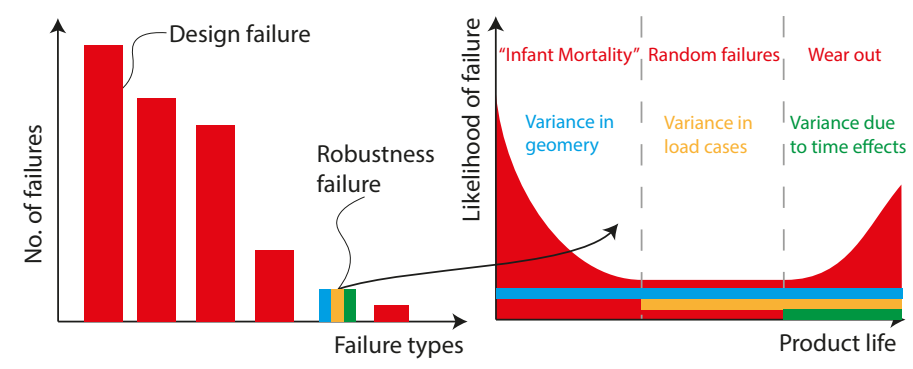

FIGURE 4. CORRELATION BETWEEN NUMBER OF FAILURES AND FAILURE TYPE

number and frequency of experienced failures [6]. If the same failure type is detected very often and caused with nominal geometry, this is referred to as a design failure, see left part of figure 4. An example could be an axle breaking for the specified load requirement. However, if a failure type is detected irregularly is it highly likely to be a robustness related failure. This type of failure can occur throughout the product life as seen in the right part of figure 4. It is theorized by the author that different types of variance could start and be dominant in the different phases of the product life. Failures related to variance in geometry would be dominant in the assembly/start of life but could occur throughout the product life. Failures related to load might occur after several uses and time effects such as material degradation might first occur closer to the end of product life. Note that the figure is caricatured and the specific starts could vary depending on design and product type.

The four robustness issue examples stated below are mainly caused by variance in geometry. However, understanding load cases and time effects can be just as important. Especially time effects on materials such as polymers where creep and relaxation occurs.

Example 1: A product has been utilizing a spring and housing design, which has had continues performance issues after launch. As the overall product design is nearly impossible to change once the highly automated production lines are established, only minor changes are achievable. The issue is attempted to be solved by ideas from the production and the design team to reduce scrap. As a result the current spring design is on version 35 .

Example 2: A new product has being developed and on paper it looked like the team had achieved the perfect design in regards to RDM (kinematics, contacts, etc.). However, when the design was produced and variance was introduced the design performed poorly and had to be cancelled due to sensitivity issues.

Example 3: A product had been in production for 7 years and every year the scrap rates were drastically higher than expected due to assembly related issues, caused by unforeseen variation in part geometries.

Example 4: A new product was being developed and the development team opted to reuse existing design features from a previous product. However, due to the change in dosing requirements the previously working thread of a rod had to be changed to a steeper angle. In the beginning no-one expected this to be an issue, as the previous design worked. After months of work using the old design as the foundation the new design was deemed unsuitable as the change in thread rise had significant impact on the device performance.

\section{Ideas for future VRDO tools and processes}

The previous sections clearly express the need for further development of tools and methods to identify and remove variation related design issues before product launch. Based on the feedback from the lead engineers and high-level decision makers this section aims to highlight how and where in the design process new potential robustness tools and methods should be applied for maximum impact. Three main solution areas were discussed; (1) Enhancement of the CAD environment to increase the awareness of variation, (2) Increased utilization of virtual simulation, e.g. combining FEA with DoE to create sensitivity awareness, (3) Data representation for management.

Figure 5 shows an example of the visual aid used to guide the conversation regarding enhancement of the CAD environment.

Half of the Lead Engineers thought enhancements to the existing CAD environment would add value if below suggestions could be achieved;

- Highlight functional surfaces

- Visualize tolerance chains between selected components

- Visualize kinematics (overconstrains)

- Include tolerances when designing

○ Visualize geometrical variation, e.g. overlay models

- Trigger warning if variation issues is detected/calculated

Interestingly enough software exists today that can aid with some of the above suggestions [7], which raises the question why are 


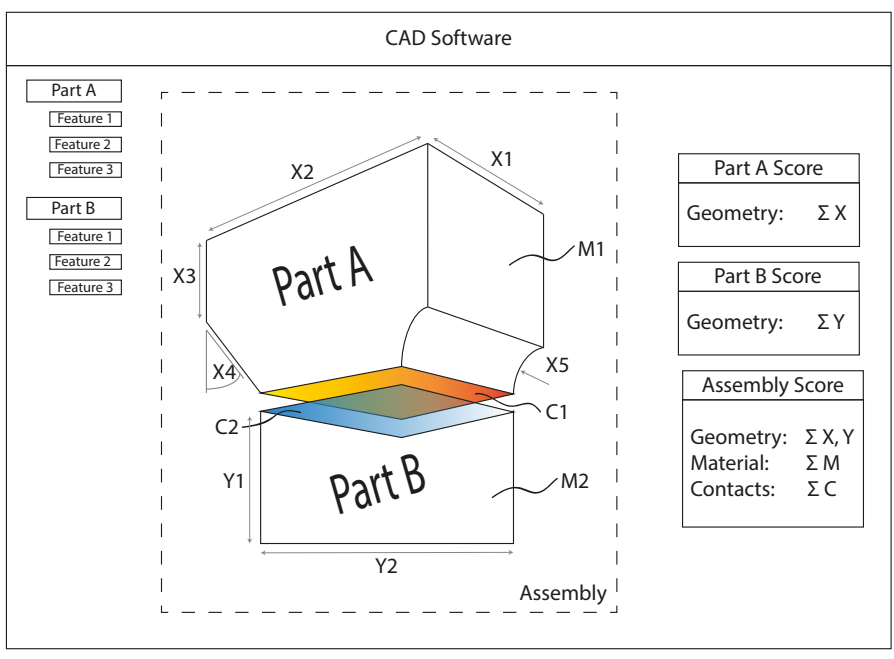

FIGURE 5. ILLUSTRATION FOR CAD ENHANCEMENT

they not used?

Figure 6 shows an example of the visual aid used to guide the conversation regarding enhancement of the FEA and DoE for robustness investigation. The top part of the figure shows a model example where the functional requirement (FR) is to minimize the deflection of the beam. The lower part of the figure shows the results from a DoE/sensitivity study utilizing FEA, where the impactful parameters are displayed in a tornado-plot. All lead engineers and high-level decision makers thought expanding the capabilities for utilizing FEA to identify robustness was key to enhance the product development, both in terms of speed to market, to increase the innovation height and patient safety. The key to success is to make this toolbox easily available for the organization and not complicate the existing workflow where time-constrains is a major concern. For simple designs a skilled engineer would be able to correlate which design parameters impacts a single functional requirement. However, in designs with multiple functional requirements the complexity increases dramatically to where a design parameter can impact FR1 positively and FR2 negatively, creating trade-off scenarios. Today sub-optimization occurs as the total overview of all these interaction effects is too complex. If sensitivity plots can be created efficiently, e.g. automate the DoE-FEA work-flow, it would enable the designers to;

- Identify sensitive relationships between functional requirements and design parameters

- Adjust the design parameter to reduce design sensitivity but exploiting non-linearities

- Re-run simulations to confirm achieved robustness

Finally, figure 7 shows an example of the visual aid used to guide the conversation regarding representation of data for the

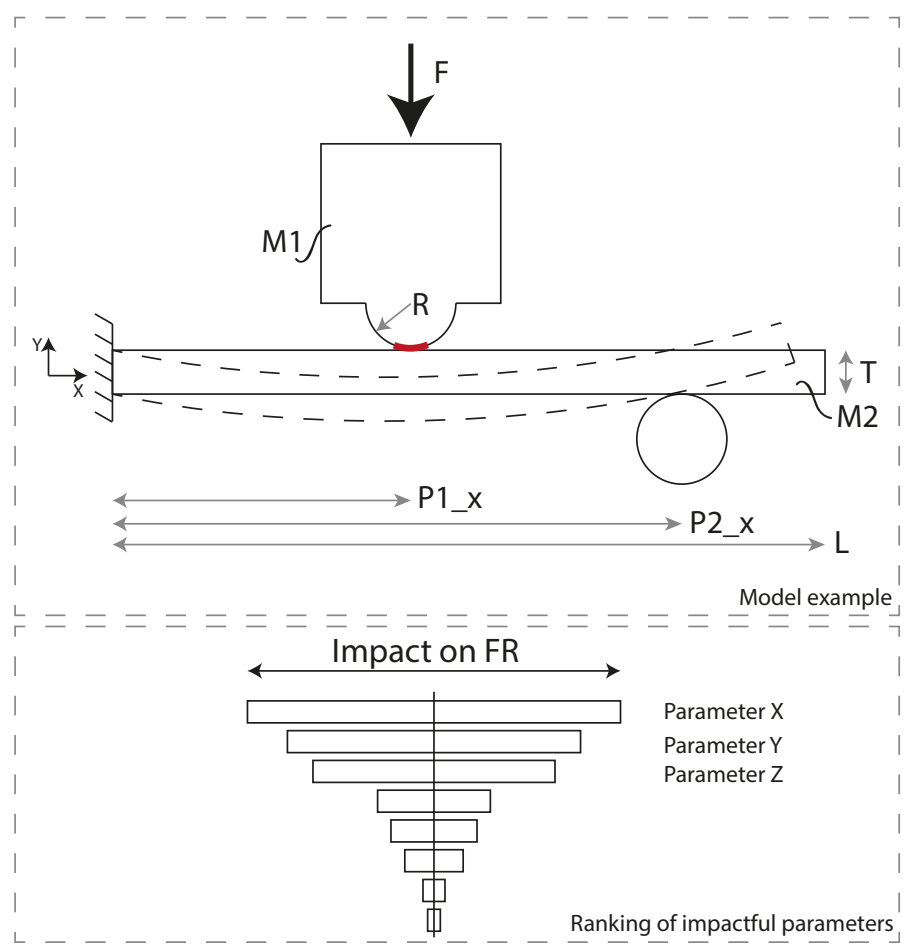

FIGURE 6. ILLUSTRATION FOR FEA AND DOE ENHANCEMENT

management. The discussion revolved around the possible future, where failure distributions could be calculated by including large scale simulation and thereby create a paradigm shift from safety factors to part per million (PPM) failures. All interviewees agreed that this would greatly enhance the confidence level of decisions. Another strong benefit would be that the world of risk management work within the same domain of PPM values.

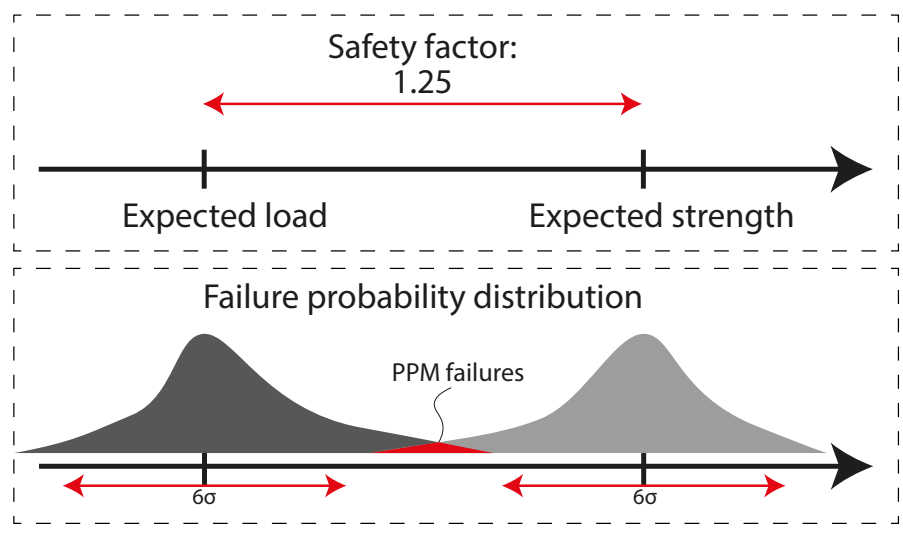

FIGURE 7. ILLUSTRATION FOR DATA REPRESENTATION FOR MANAGEMENT

Copyright (C) 2019 by ASME 


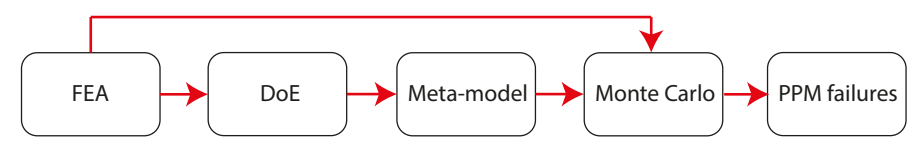

FIGURE 8. ADVANCED ROBUSTNESS IDENTIFICATION WORK-FLOW

Achieving these results would require additional expansion of the FEA and RD toolbox. Discussed solution can be visualized in figure 8. Depending on the complexity of the simulation or the available computing power, different work-flows would be feasible. Realistically the lower linear work-flow is expected, where no steps can be skipped. Creating a meta-model (an approximation of a higher-fidelity model) removes the large computational strain as the Monte-Carlo models can be solved analytically. If Monte-Carlo should be achieved directly, a large amount of numerical simulations had to be run. This would require an extreme amount of available computational power. However, with the focus on cloud-computing this might be possible in the future.

\section{Industrial barriers for further implementation of VRDO}

The previous sections has covered existing decisions tools and suggested functionalities for the enhanced VRDO. The following section to wishes to present a list of discovered barriers to achieve the suggested implementation of VRDO in an industrial context. Each barrier is categorized as either; A solution exists, either in form of software, training, etc. or that a residual issue remains to be solved. The parentheses $(\checkmark)$ expresses that more investigation is required. The hypotheses and results are based on the gathered interview material, literature review in the fields of FEA and RDM, and software investigation presented in an anectdotal form.

The key technical barrier from table 2 is software availability. To enable efficient use of VRDO commercially the software has to be available from third-party suppliers. The complexity is simply too great for in-house development. The shortlist presented in table 4 clearly shows that several companies are starting to supply software supporting VRDO. The list ranges from major market players like Dassault to smaller "start-up" companies like CORTIME. Each are aiming to differentiate their product to gain a specific market segment. Similar to CAD software, VRDO software is highly dependent on the user requirements. This is, for example, one of the reasons why Dynardo optiSLang has a chance to compete with ANSYS, by utilizing their solver/platform, while ANSYS provides a similar product. Depending on the supplier differentiation is made between easy to use applications versus loss of simulation accuracy or control. The second key technical challenge is the CPU-time related to DoE. Already today an advanced simulation can take multiple hours/days to compute. Introducing DoE will greatly increase the already critical time parameters in projects. Further inves-

\section{TECHNICAL BARRIERS}

\begin{tabular}{lc}
\hline Hypothesis & Solution \\
\hline No commercial software enables VRDO \\
Tools are too complicated \\
Massive increase in CPU-time due to DoE \\
$\begin{array}{l}\text { Required tools exists in different soft- } \\
\text { ware solutions which doesn't effectively } \\
\text { exchange information }\end{array}$ \\
$\begin{array}{l}\text { Unable to work on model design and sim- } \\
\text { ulation in parallel }\end{array}$ \\
$\begin{array}{l}\text { No effective methods for automating ad- } \\
\text { vanced studies }\end{array}$ \\
\hline
\end{tabular}

TABLE 2. DISCOVERED TECHNICAL BARRIERS

tigation is required to define which type of fractional factorial designs is best suited for different types of simulations. An alternative approach could be to sacrifice some of the simulation accuracy to gain lower CPU-time, while achieving a significant insight into the relative sensitivity between functional requirements and design parameters. Sacrificing accuracy might not normally be considered excellent FEA practice, but it might find its' uses in VRDO. The software companies provide solutions to the remaining barriers in their product material, however, as the author is yet to confirm this further investigation is required.

The discovered barriers related to knowledge, seen in table 3 are the least problematic doing this investigation. This can be positively affected by the frontier position of the industrial company interviewed, but also the software company expresses an increased interest in their product. Nevertheless, it confirms the importance of the investigated research area and that all efforts to enhance VRDO would be rewarded by the industry.

Table 5 contains a long list of practical barriers before efficient commercial VRDO can be achieved. The first hypothesis is both considered a technical and practical, which needs to be solved by either increasing allowed project time, increase available computing power or reduce the computing time per simulation. The second hypothesis relates to cost. Increased competition could potentially reduce the cost of software licence. Alternatively companies must accept the cost to increase the design reliability. Third and fourth are related to either lack of training or missing software capabilities. The remaining are all related to training and usage of the new software capabilities. 


\section{KNOWLEDGE BARRIERS}

\begin{tabular}{l}
\hline Hypothesis \\
\hline No need for extended virtual development \\
(DoE/Sensitivity/Monte-Carlo) \\
Project teams are unaware of the tools \\
available (no active "pull") \\
Other parts of the robust design process \\
would create more value to enhance
\end{tabular}

TABLE 3. DISCOVERED KNOWLEDGE BARRIERS

\begin{tabular}{l|l} 
Software company & Connected with \\
\hline \hline CORTIME & SOLIDWORKS \\
\hline Dynardo optiSLang & $\begin{array}{l}\text { ANSYS, Abaqus, CATIA, Creo, } \\
\text { Nastran, SimulationX }\end{array}$ \\
\hline Esteco & $\begin{array}{l}\text { ANSYS, Abaqus, Creo, } \\
\text { Siemens, SOLIDWORKS, +12 } \\
\text { more }\end{array}$ \\
\hline Caeses & $\begin{array}{l}\text { CATIA, NX, Pro/E, SOLID- } \\
\text { WORKS }\end{array}$ \\
\hline Siemens HEEDS & $\begin{array}{l}\text { ANSYS, Abaqus, Creo, } \\
\text { SOLIDWORKS, +30 more }\end{array}$ \\
\hline Noesis & $\begin{array}{l}\text { CATIA, ANSYS, Abaqus, Creo, } \\
\text { SOLLIDWORKS, +10 more }\end{array}$ \\
\hline OptiY & $\begin{array}{l}\text { CATIA, ANSYS, Creo, SOLID- } \\
\text { WORKS, +10 more }\end{array}$ \\
\hline ANSYS DesignXplorer & Own platform \\
\hline \hline Dassualt 3DExperience & Own platform \\
\hline \hline Oltair HyperWorks & Own platform \\
\hline
\end{tabular}

TABLE 4. SOFTWARE COMPANIES PROVIDING ROBUST DESIGN OPTIMIZATION SOLUTIONS

\section{DISCUSSION}

In this paper several barriers for implementing VRDO has been categorized and presented. The types of barriers discovered is a reflection of the data gathered during the interviews with the two selected companies. Since VRDO has the potential to assist design engineers across companies and industries, interesting work lies ahead to investigate whether the identified barriers are generic across different industries and companies. The re-
PRACTICAL BARRIERS

Hypothesis
Simulations can exceed the time-
constrains
More tools increase the cost by either more
specialists and/or software licences
Efficient design version control is difficult
Traceability of why design changes were
implemented
Steep learning curve which lowers the will-
ingness to implement
Limited specialists available
Parameterization of CAD models are com-
pleted late in the design process
Advanced simulations can increase poten-
tial mistrust in the results
Simulation output is difficult to convert
into design changes for the designer
Advanced results are difficult to under-
stand for high-level decision makers (de-
cision in a 1-hr meeting)

\section{TABLE 5. INVESTIGATED PRACTICAL BARRIERS}

quest for VRDO and types of barriers could vary significantly depending on the industry due to multiple factors like;

- Consequence of product failure

- Competition in the market

- Available resources and competencies in the company

- Design traditions

$\circ$ Etc.

However, if barriers exist in the medical industry, it could be expected that other industries face the same or more. Additionally, each company and industry must be aware of the types of impactful variances; both in terms of size but also their distributions. As stated in [8, p.-1032]; most of the uncertain quantities appearing in engineering systems are non-Gaussian in nature (e.g. material, geometric properties, wind, seismic loads). However, the Gaussian assumption is often used due to its simplicity and the lack of relevant experimental data. This raises an interesting question on how to create and maintain variance libraries required as input for DoE, sensitivity and Monte-Carlo studies. Assuming that fairly accurate variance sizes and distri- 
butions can be provided, the second process step is deciding the type of method to be applied. It is the authors' expectation that initially for DoE only a small number of runs will be achievable due to the available computing power. Depending on the design complexity and the number of design parameters to be included in the investigation, different techniques should be applied, e.g. fractional factorial, Plackett-Burman or supersaturated designs. If the computing power remains an issue, due to the complexity of the simulation, even more aggressive approaches could be applied, such as sacrificing simulation accuracy by simplifying the geometry or mesh. If multiple functional requirements and design parameters are investigated the expected amount of data will be immense, especially if trade-off scenarios occur. This is an interesting topic to further investigate and understand how the data is best represented for the designers to perform the geometrical modifications. To achieve failure distributions, the MonteCarlo method must be applied. If performed directly by FEA a vast amount of simulations are required; several times more than what is required for a DoE. Instead, it is suggested to create meta-models where the accuracy is sacrificed, but analytical Monte-Carlo solutions are enabled. Furthermore, performing above types of simulations only once will not suffice. Once the initial results are produced, optimization of the design parameters can be obtained. To validate the achieved robustness, simulations will once again have to be performed. For this automation of the process is required as this might occur multiple times during a design process. So-called process apps are available in some commercial software packagaes for this purpose and they can be configured to import modified geometry, apply the finite element analysis and automatically re-run the simulation and compare results. Configurability and easy of use of such apps require further investigation.

\section{CONCLUSION}

The purpose of this paper was to investigate barriers for implementing virtual robust design optimization in an industrial context. The material presented concludes on the top- 3 barriers that; (1) Commercial software exists that can enable companies to perform VRDO, (2) Incorporating VRDO successfully will require a substantial practical effort and (3) The currently available computing power can constrain the size of possible virtual studies which will require further investigation. Additionally, all interviewed parties confirm that with a successful implementation and use of VRDO it would enable a more efficient design process which can lead to;

- Increased design decision certainty

- More robust designs

- More innovation

- Lower production costs, i.e. fewer design iterations and latestage changes [9]
- Increase speed to market

\section{ACKNOWLEDGMENT}

The authors would like to thank Novo Nordisk R\&D STAR Fellowship Programme and the Innovation Fund for supporting the research project.

\section{REFERENCES}

[1] Coleman, P., 2012. "CRESCENDO - Collaborative and Robust Engineering using Simulation Capability Enabling Next Design Optimisation". TRIMIS - Transport Research and Innovation Monitoring and Information System.

[2] Thornton, A. C., 2004. Variation Risk Management: Focusing Quality Improvements in Product Development and Production. Wiley.

[3] Hasenkamp, T., Arvidsson, M., and Gremyr, I., 2009. "A review of practices for robust design methodology". Journal of Engineering Design, 20(6), pp. 645-657.

[4] Lucienne T.M. Blessing, A. C., 2009. DRM, a Design Research Methodology. Springer.

[5] Pahl, G, Beitz, W., Feldhusen, J., Grote, K., 2007. Engineering Design, third ed. Springer.

[6] Krogstie, L., Ebro, M., and Howard, T. J., 2015. "How to implement and apply robust design: insights from industrial practice". Total Quality Management and Business Excellence, 26(11-12), pp. 1387-1405.

[7] Sigurdarson, N., Eifler, T., and Ebro, M., 2018. "The Applicability of CAT tools in industry-boundaries and challenges in tolerance engineering practice observed in a medical device company”. Procedia CIRP, 75, pp. 261-266.

[8] Stefanou, G., 2009. "The stochastic finite element method: Past, present and future". Computer Methods in Applied Mechanics and Engineering, 198(9-12), pp. 1031-1051.

[9] Folkestad, J., and Johnson, R., 2001. "Resolving the conflict between design and manufacturing: Integrated Rapid Prototyping and Rapid Tooling (IRPRT)". Journal of Industrial Technology, 17(4). 